\title{
The STEREO IMPACT Suprathermal Electron (STE) Instrument
}

\author{
R.P. Lin · D.W. Curtis • D.E. Larson • J.G. Luhmann • \\ S.E. McBride • M.R. Maier - T. Moreau • C.S. Tindall • \\ P. Turin · Linghua Wang
}

Received: 27 October 2006 / Accepted: 5 February 2008 / Published online: 13 March 2008

(C) Springer Science+Business Media B.V. 2008

\begin{abstract}
The Suprathermal Electron (STE) instrument, part of the IMPACT investigation on both spacecraft of NASA's STEREO mission, is designed to measure electrons from $\sim 2$ to $\sim 100 \mathrm{keV}$. This is the primary energy range for impulsive electron $/{ }^{3} \mathrm{He}$-rich energetic particle events that are the most frequently occurring transient particle emissions from the Sun, for the electrons that generate solar type III radio emission, for the shock accelerated electrons that produce type II radio emission, and for the superhalo electrons (whose origin is unknown) that are present in the interplanetary medium even during the quietest times. These electrons are ideal for tracing heliospheric magnetic field lines back to their source regions on the Sun and for determining field line lengths, thus probing the structure of interplanetary coronal mass ejections (ICMEs) and of the ambient inner heliosphere. STE utilizes arrays of small, passively cooled thin window silicon semiconductor detectors, coupled to state-of-the-art pulse-reset front-end electronics, to detect electrons down to $\sim 2 \mathrm{keV}$ with about 2 orders of magnitude increase in sensitivity over previous sensors at energies below $\sim 20 \mathrm{keV}$. STE provides energy resolution of $\Delta E / E \sim 10-25 \%$ and the angular resolution of $\sim 20^{\circ}$ over two oppositely directed $\sim 80^{\circ} \times 80^{\circ}$ fields of view centered on the nominal Parker spiral field direction.
\end{abstract}

Keywords Solar energetic particles · Energetic particle detectors $\cdot$ STEREO mission · Suprathermal electrons $\cdot$ Silicon detectors

R.P. Lin $(\bowtie) \cdot$ L. Wang

Physics Department \& Space Sciences Laboratory, University of California, Berkeley, CA 94720-7450, USA

e-mail: rlin@ssl.berkeley.edu

D.W. Curtis · D.E. Larson · J.G. Luhmann · S.E. McBride · T. Moreau · P. Turin

Space Sciences Laboratory, University of California, Berkeley, CA 94720-7450, USA

M.R. Maier · C.S. Tindall

Lawrence Berkeley National Laboratory, Berkeley, CA 94720, USA 


\section{Introduction}

The SupraThermal Electron (STE) instrument is part of the IMPACT (In-situ Measurements of Particles And CME Transients) investigation (see Luhmann et al. 2007, for overview), one of the four instrument packages on both spacecraft of NASA's STEREO (Solar TERrestrial RElations Observatory) mission. STE is designed to measure the $\sim 2-100 \mathrm{keV}$ suprathermal "superhalo" electrons (Fig. 18, panels a \& b, of Luhmann et al. 2007) that was discovered by Wind spacecraft observations to be present in the interplanetary medium (IPM) near $1 \mathrm{AU}$, even during the quietest times (Lin 1997). They are the dominant particle species in impulsive solar energetic particle (SEP) events, the most frequently occurring transient solar emissions. These fast electrons with small gyro-diameters are ideal tracers of solarheliospheric magnetic field connections, since the X-ray and radio emission they generate at the Sun and in the IPM can be detected remotely. Furthermore, they suffer significant energy losses in traversing the corona, so their energy spectra can provide information on their source height. Thus, they are powerful probes of particle acceleration and escape from the Sun, and of solar-heliospheric magnetic structure and connectivity, both for transients (ICMEs) and the quiet heliosphere.

Previously, measurements of particles below $\sim 20-30 \mathrm{keV}$ energies were made by electrostatic analyzers (ESAs) with microchannel plate detectors (e.g., the EESA and PESA (electron and proton) detectors of the 3D plasma \& Energetic Particles investigation on the Wind spacecraft (Lin et al. 1995) and the SWEA (Solar Wind Electron Analyzer) detector of IMPACT [see Sauvaud et al. 2007]). The fluxes of suprathermal electrons, however, are typically many orders of magnitude lower than the solar wind halo and strahl electrons (see Fig. 18, panels a \& b, of Luhmann et al. 2007). STE is designed to provide about 2 orders of magnitude increase in sensitivity over previous measurements in the $\sim 2$ to $20-30 \mathrm{keV}$ energy range, by extending the range of silicon semiconductor detectors (SSDs) for particle detection down to $\sim 2 \mathrm{keV}$. STE's sensitivity and resolution in energy and angle enables the detailed study of solar and interplanetary particle acceleration, escape, and propagation; of the structure and solar connectivity of the IPM, in particular, ICMEs; and of the waveparticle processes (together with the STEREO waves (SWAVES) instrument (see Kaiser et al. 2007) that produce solar type III and type II radio bursts.

\section{Scientific Objectives}

STE measurements will directly address many of the prime objectives of the STEREO mission. It will:

- Probe particle acceleration near the Sun in both impulsive and gradual SEP events.

- Identify the magnetic footpoints and determine the length of field lines of ICMEs.

- Probe the in situ acceleration of electrons by ICME shock waves,

- Study the plasma physics of the wave-particle interactions that generate type II and III radio emission.

- Identify the source (presently unknown) of the superhalo $(\sim 1-100 \mathrm{keV})$ electrons that are always present in the interplanetary medium.

Solar impulsive electron events were first observed above $\sim 40 \mathrm{keV}$ and later detected down to keV energies (see Lin 1985 for review), and even to $\sim 0.1 \mathrm{keV}$ (Gosling et al. 2003), They are almost always accompanied by an interplanetary type III solar radio burst, and often by low-energy, tens of $\mathrm{keV}$ to $\sim \mathrm{MeV} /$ nucleon ions highly enriched in ${ }^{3} \mathrm{He}$ (Reames 
et al. 1985), forming the class of "impulsive" SEP events. Over 100 events/month occur over the whole Sun near solar maximum above pre-STE detection thresholds, making these the most frequent transient energetic particle emissions from the Sun (Lin 1985).

Using WIND observations, Krucker et al. (1999) found the injection of the $>25 \mathrm{keV}$ electrons were often delayed by an order of $\sim 10$ minutes after the type III radio bursts at the Sun, and suggested the delays may be related to propagation of large-scale coronal transient (EIT or Moreton) waves across the Sun. Haggerty and Roelof (2002) found a median delay of 10 min for 38-315 keV electrons; Simnett et al. (2002) suggested acceleration by shock waves associated with coronal mass ejections (CMEs). Maia and Pick (2004) proposed that these events are related to the coronal magnetic restructuring in the aftermath of CMEs, while Cane and Erickson (2003) argue from radio observations that the delays are due to propagation effects in the IPM. Comparisons to SOHO/LASCO coronagraph and EIT observations indicate the delayed high energy injections may be related to jets or narrow CMEs (Wang et al. 2006b).

More recently, Wang et al. (2006a) was able to derive the injection profile of electrons at the Sun for three extremely scatter-free impulsive electron events. They found two distinct injections: one at low energies $(\sim 0.4$ to $\sim 10 \mathrm{keV})$ that begins minutes before the type III burst at the Sun and lasts for 50-300 minutes, and one at high energies ( $>\sim 13$ to $300 \mathrm{keV}$ ) that is delayed by $\sim 10$ minutes from the type III burst and lasts for $\sim 10-30$ minutes. Thus, the source of the type III radio burst can be identified with the low-energy electrons, consistent with the detection of Langmuir waves in situ when the $\sim 1-10 \mathrm{keV}$ electrons arrive near 1 AU (Ergun et al. 1998).

STE's extremely high sensitivity for suprathermal electrons will provide many more impulsive solar electron events for study than previous missions. Electron injection profiles, energy spectra, and pitch angle distributions in impulsive and gradual SEP events will be determined from measurements with STE. Simultaneous identical measurements by the two STEREO spacecraft as they move away from each other will provide information on the longitudinal extent of the cone of field lines populated by these events. Comparison of these in situ electron observations at $\sim 1$ AU with RHESSI (Ramaty High Energy Solar Spectroscopic Imager mission, see Lin et al. 2002) measurements of the spectrum, temporal profile, and imaging of the bremsstrahlung X-rays produced by these energy electrons at the Sun will provide detailed information about electron acceleration and escape processes in impulsive and gradual SEP events.

Electrons in impulsive SEP events provide ideal tracers of magnetic field connection from the Sun into the heliosphere. They can be located at the Sun through their bremsstrahlung X-ray emission (imaged by RHESSI), tracked as they travel along the magnetic field lines through the interplanetary medium by the type III radio bursts that they generate (with the triangulation by the SWAVES instruments on the two STEREO spacecraft), and detected in situ by the STE instruments on the two spacecraft. By analyzing the velocity dispersion of these impulsively accelerated electrons, the field line length can be obtained in both ICMEs, where the lengths can be several times the typical Parker spiral length at the outer edge of the twisted field of a magnetic flux rope (see Fig. 2 in Luhmann et al. 2007 [from Larson et al. 1997]), and in the quiet interplanetary medium.

These electron diagnostics of ICME magnetic field line properties, obtained from both STEREO spacecraft, can be compared with the stereo imaging of the CME/ICMEs from the SECCHI (Sun Earth Connection Coronal and Heliospheric Investigation) instruments, and to the radio imaging from SWAVES instruments on STEREO. The extremely high sensitivity of STE, coupled with the time resolution (2 sec at $20 \mathrm{keV}$ ) with which the anisotropy measurements are made, provides the best opportunity to date to search for the signatures 
of the closing down of interplanetary field connections to the Sun, to evaluate the balance between opening and closing flux in ICME transients, and to determine whether the slow solar wind (and by inference, the coronal streamer belt) is an important participant in the flux balance problem.

The SWAVES instrument will track CME-driven shocks and flare electrons as they propagate away from the Sun, using type II and type III radio bursts as a proxy. This tracking, however, depends on the beam pattern of the radio burst, which is specific to the radio emission mechanism. It is believed that plasma waves are generated at $f_{\text {pe }}$, the electron plasma frequency, by suprathermal $\sim 2-20 \mathrm{keV}$ electrons escaping from the Sun (see Lin et al. 1986). Wind observes elliptically polarized plasma waves at $1 f_{\mathrm{pe}}$ in the source of these bursts, where longitudinally polarized Langmuir waves were expected (Bale et al. 1998). These plasma waves then interact, in a poorly understood way, to produce radio emissions at $f_{\mathrm{pe}}$ and $2 f_{\mathrm{pe}}$. How the radio emissions depend on the electron distribution and ambient plasma characteristics is unknown. STE will provide electron measurements to compare with SWAVES radio and plasma wave measurements.

Recently, Wind provided the first observation of the Type II electron source in situ (Bale et al. 1999). The radio emission is observed to come from near quasi-perpendicular connections to the ICME-driven shock, while the source shock-accelerated electrons show the shock surface is highly structured, giving rise to multiple emission sites. As type II and III radio source regions pass over the two STEREO spacecraft, the IMPACT and SWAVES instruments will make coordinated burst observations of the suprathermal electrons and plasma waves. The exceptional sensitivity, temporal, and energy resolution of STE, when coupled with SWAVES 3 axis electric and 1 axis magnetic waveforms, should lead to advances in the understanding of the type III and type II radio emission processes. Together, SWAVES and STE will further resolve the Type II source nature, allowing us to better infer shock structure from remote particle and radio measurements.

Above $\sim 2 \mathrm{keV}$ the "superhalo" electron component (Fig. 18, panels a \& b, of Luhmann et al. 2007) that is present even during the quietest times (Lin 1997) typically extends up to $>100 \mathrm{keV}$. Its origin is presently unknown. These electrons could be related to the solar wind acceleration process, or result from continuous acceleration by micro/nanoflares in the solar atmosphere, or come from interplanetary acceleration processes similar to those likely responsible for the suprathermal ions. As mentioned earlier, the impulsive acceleration of electrons to $\sim 1$ to $>\sim 100 \mathrm{keV}$ energies is the most common solar particle acceleration process. Detailed high sensitivity measurements by STE of the superhalo, including its spectral, angular, temporal and spatial properties, are needed to determine its origin.

\section{Instrument Overview}

STE is a new instrument designed to provide geometry factor times observing time product about two orders of magnitude larger than previous detectors (electrostatic analyzers) in the $\sim 2$ to $20 \mathrm{keV}$ energy range, with much lower background. Electrostatic analyzers such as the very large EESA-H sensor ( $\sim 20 \mathrm{~cm}$ diam, $\sim 3 \mathrm{~kg}, \sim 3 \mathrm{~W}$ ) of the 3D Plasma \& Energetic Particle instrument (Lin et al. 1995) on the Wind spacecraft measure one energy band at a time. EESA-H has $\Delta E / E=\sim 0.2$ so $\sim 14$ contiguous energy steps covered 2-20 keV, a duty cycle of $\sim 7 \%$ at a given energy. STE utilizes silicon semiconductor detectors (SSDs) that measure all energies simultaneously (100\% duty cycle). Up to now the SSDs used for particle detection have had relatively high capacitance and leakage currents, resulting in electronic noise thresholds of $>\sim 15 \mathrm{keV}$, and thick window dead layers that further increase the minimum 


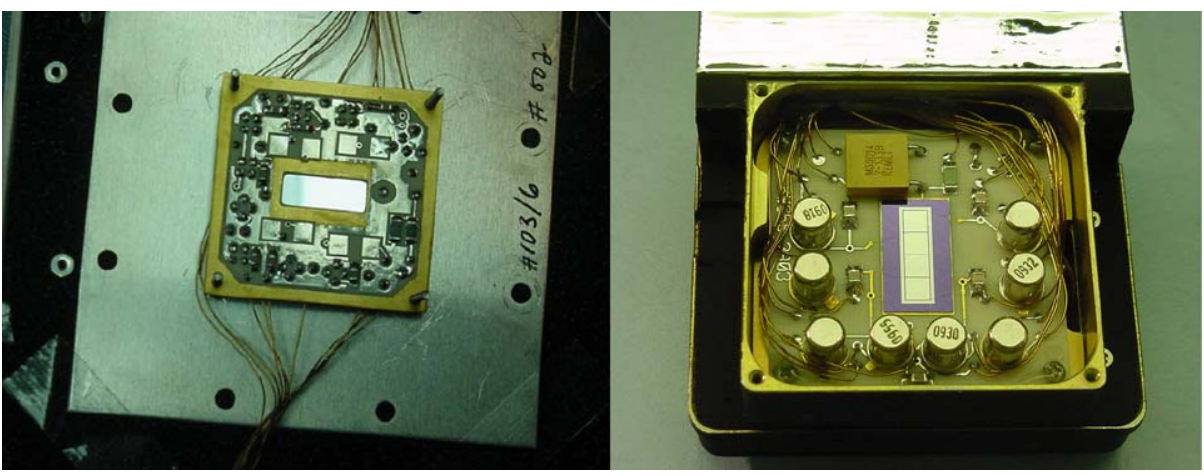

Fig. 1 Detector board front side (left) and back side shown in housing (right)

Fig. 2 Detector pixel fields of view (FOV), maximum extent, for STE_D; STE-U has a $70^{\circ} \times 70^{\circ}$ FOV. The FWHM FOVs are $\sim 20^{\circ} \times 70^{\circ}$ for each pixel

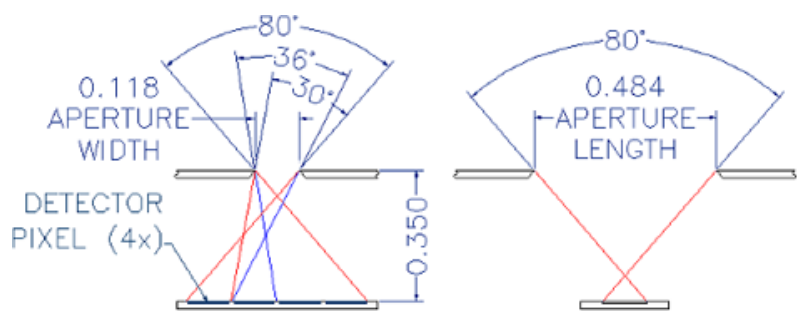

energy detectable to $\sim 20-30 \mathrm{keV}$ for electrons and ions. STE consists of arrays of small, low capacitance, cooled SSDs (Fig. 1), fabricated with an unusually thin window dead layer (Tindall et al. 2008), so $<\sim 2 \mathrm{keV}$ electrons can penetrate and be detected. The SSDs are surrounded by a guard ring and passively cooled to $\sim-30$ to $-90^{\circ} \mathrm{C}$ to minimize leakage current, and coupled to state-of-the-art, cooled FET, pulse-reset preamp-shaping electronics to obtain an electronic threshold of $\sim 1.5 \mathrm{keV}$.

STE consists of two arrays, STE-U and STE-D, each with four SSDs in a row on a single silicon wafer, looking through a rectangular opening (Fig. 2) that provides a $\sim 20^{\circ} \times \sim 80^{\circ}$ field of view (FOV) for each SSD (with the $80^{\circ}\left( \pm 40^{\circ}\right)$ direction perpendicular to the ecliptic). Adjacent FOVs are offset from each other by $\sim 20^{\circ}$ for pitch angle resolution of $\sim 20^{\circ}$ FWHM. The two arrays each have total nominal FOV of $\sim 80^{\circ} \times 80^{\circ}$ (STE-D), centered on the nominal Parker spiral magnetic field direction, one array facing toward (STE-U) and the other (STE-D) away from the Sun. Since the thin-window SSDs are very sensitive to light, carefully designed sun shades are needed to prevent stray light and glint from the spacecraft and booms from entering the aperture. Table 1 summarizes the measurement parameters and instrument characteristics for STE.

STE has a total geometric factor of $\sim 0.2 \mathrm{~cm}^{2}$ ster vs 0.05 for Wind/EESA-H, a $100 \%$ duty cycle vs $7 \%$, for a factor of $\sim 60$ gain in effective signal. EESA-H used an anticoincidence shield to reject penetrating high-energy particle background, but even so the count rate above $\sim 2 \mathrm{keV}$ at quiet times was $\sim 30 \mathrm{c} / \mathrm{s}$, dominated by intrinsic background counts of the very large microchannel plate detector. The primary background at $2-20 \mathrm{keV}$ for the STE detectors is expected to be diffuse sky X-rays ( $\sim 1 \mathrm{c} / \mathrm{s}$ per pixel), but this is much lower than the anticipated electron superhalo count rate ( $>\sim 5 \mathrm{c} / \mathrm{s}$ per pixel). Penetrating particles such as cosmic rays and high energy SEPs leave more than $200 \mathrm{keV}$ (minimum ionizing) and are rejected. 
Table 1 STEREO IMPACT Suprathermal Electron (STE) instrument

A. Measurement parameters

\begin{tabular}{lllll}
\hline Detector & $\begin{array}{l}\text { Particle \& } \\
\text { energy range }\end{array}$ & $\begin{array}{l}\text { Geometric } \\
\text { factor }\end{array}$ & Field of view ${ }^{2}$ & Dynamic range \\
\hline STE-U & $\begin{array}{l}2-100 \mathrm{keV} \\
\text { electrons }\end{array}$ & $0.08 \mathrm{~cm}^{2}$ ster & $\begin{array}{l}70^{\circ} \times 70^{\circ} \\
\text { centered } 45^{\circ} \\
\text { from Sun } \\
80^{\circ} \times 80^{\circ}\end{array}$ & $\begin{array}{l}\sim 0.1-10^{6} \\
\left(\mathrm{~cm}^{2} \mathrm{ster} \mathrm{s}^{-1}\right.\end{array}$ \\
STE-D & $\begin{array}{l}\text { centered } 225^{\circ} \\
\text { from Sun }\end{array}$ & $\begin{array}{l}\sim 0.1-10^{6} \\
\left(\mathrm{~cm}^{2} \mathrm{ster} \mathrm{s}^{-1}\right.\end{array}$ \\
\hline
\end{tabular}

${ }^{1}$ Energy resolution $\Delta E / E$ is $\sim 1 \mathrm{keV}$ at low energies to $\Delta E / E \sim 0.2$ at high energies

${ }^{2}$ Each of the four detectors has a $\sim 17^{\circ} \times 70^{\circ}$ FWHM FOV with $\sim 18-19^{\circ}$ for STE-D spacing between detectors

B. Instrument characteristics

\begin{tabular}{|c|c|c|c|c|}
\hline & Mass $^{3}$ & Power 4 & Volume & Bit rate \\
\hline STE-U & $0.4 \mathrm{~kg}$ & 0.5 watts & & \\
\hline STE-D & $0.4 \mathrm{~kg}$ & 0.5 watts & & \\
\hline Total & $0.8 \mathrm{~kg}$ & 1.0 watts & & $230 \mathrm{bps}$ \\
\hline
\end{tabular}

${ }^{3}$ Does not include bias supply and other electronics in IDPU

${ }^{4}$ Does not include part of SWEA or IDPU used for STE, or conversion efficiency

Low energy ions (protons $>\sim 4 \mathrm{keV}, \mathrm{He}>\sim 8 \mathrm{keV}$, etc.), however, can penetrate the window and stop. Energetic neutral atoms (ENAs) or molecules, if present, will be detected as ions, since they will be ionized upon passing through the detector window. The STE FOV excludes solar wind ions, but pickup ions will fill a shell in velocity space of radius $\sim V_{\text {sw }}$ centered on the solar wind (Mobius et al. 1998). For typical $V_{\text {sw }} \sim 400 \mathrm{~km} / \mathrm{s}$, pickup protons will be below threshold, but pickup He are expected to be detected with typical fluxes of $\sim 1-2 \times 10^{3}\left(\mathrm{~cm}^{2} \mathrm{~s} \text { ster } \mathrm{keV}\right)^{-1}$, from directions within $\sim 45^{\circ}$ of the solar wind. The SSD whose FOV is closest to the solar Wind will see He count rates of a few hundred per second (an interesting measurement in itself), while the count rates of the other SSDs should be dominated by superhalo electrons. Of course, at higher $V_{\mathrm{sw}}$ more pickup ions will be detected, in more SSDs.

For detection of impulsive electron events (defined somewhat arbitrarily as an 5 sigma increase in 5 minutes), STE should have greater than two orders of magnitude more sensitivity than EESA-H. Thus, far more events will be detected. At the same time STE requires significantly less resources than EESA-H (Table 1).

The STE sensors (including detector, FET, enclosure, door) are $40 \mathrm{~g}$ each. The STE-U preamp and enclosure is $360 \mathrm{~g}$. The STE-U electronics card (in the IDPU) is $300 \mathrm{~g}$. STE-U shares the processor $(260 \mathrm{~g})$ and LVPS ( $280 \mathrm{~g}$ ) of the IDPU. The IDPU chassis is $690 \mathrm{~g}$. The STE-D preamp and electronics is included in the SWEA/STE-D package. The combined SWEA/STE-D electronics, including shared LVPS, shared digital interface, shared chassis, and STE preamp and electronics (but excluding STE-D sensor, SWEA sensor, SWEA preamps and HVPS) weighs $960 \mathrm{~g}$. STE-D also shares the IDPU processor. Thus, a standalone STE unit without processor or LVPS would be $\sim 700 \mathrm{~g}$. 
Each STE unit dissipates $<100 \mathrm{~mW}$ in the sensor, $\sim 200 \mathrm{~mW}$ in the preamp, and $\sim 290 \mathrm{~mW}$ in the remaining electronics (excluding processor and LVPS). So the total for a stand-alone STE is $\sim 600 \mathrm{~mW}$, excluding processor and LVPS.

\section{Silicon Semiconductor Detectors (SSDs)}

STE's SSDs have very thin entrance contacts fabricated through a processing technique that employs a thick, doped polysilicon layer on the wafer backside to getter impurities during the high temperature processing steps. This helps to maintain the material resistivity at values greater than $10 \mathrm{k} \Omega-\mathrm{cm}$ throughout the process and minimizes the incorporation of deep levels within the active area of the device that lead to increased leakage current (Holland 1989). In order to form the thin contact, near the end of the process, the one micron thick gettering layer was removed either by chemical etching or chemical-mechanical polishing (CMP). A 100 to $200 \AA$ thick, doped polysilicon layer was then deposited on the entrance side of the detectors to form the $n+$ contact (Holland et al. 1997). Although the SSDs are well shielded from stray light, a $200 \AA$ thick layer of aluminum was deposited over the doped-poly silicon contact to further reduce the SSDs' sensitivity to light.

For STE, $1 \times 4$ linear arrays of $3 \mathrm{~mm} \times 3 \mathrm{~mm}$ SSDs (Fig. 1) were fabricated on $315 \mu \mathrm{m}$ thick, high resistivity $(\sim 10 \mathrm{k} \Omega-\mathrm{cm}),\langle 100\rangle$ n-type silicon substrates, with a multiple guard ring structure to gradually drop the bias voltage and to take up the surface leakage current. The SSDs are operated fully depleted, so that the electric field extended to the back contact. Leakage currents are typically on the order of a few hundred picoamps per square centimeter at room temperature. The residual impurity level was found to be between $3 \cdot 10^{11}$ and $4 \cdot 10^{11} \mathrm{~cm}^{-3}$. The SSD elements are wirebonded to the input low noise FET of a chargesensitive preamplifier and connected to amplification, shaping and digitizing electronics.

\section{Electronics}

The Analog Electronics start with a pulse-reset charge sensitive preamplifier. The lack of feedback resistor means its contribution to the electronic noise is not present-an important source at these noise levels (450-600 eV FWHM). Additionally, it is very effective in removing the charge resulting from an extremely high energy event captured in the detector. Because of the close physical proximity of the four detectors in the array, however, a reset in a single detector will generate a signal in the other three, resulting in a dead time in all four detectors.

The preamplifier input FETs and reset transistors are mounted on a small low leakage printed circuit board very close to the SSDs (Fig. 1) so stray capacitance doesn't add electronic noise to the system, and the FETs are passively cooled along with the SSDs to minimize electronic noise. The FETs are dual gate devices chosen for their superior noise performance. The SSD/FET circuit board is connected to the rest of the preamp electronics through manganin wires to provide adequate thermal isolation. The detector/FET board also includes a thermometer, high voltage filtering, bypassing of the reset circuitry and the feedback capacitors for the charge sensitive amplifiers. A test pulser signal modulates the high voltage on the SSDs to provide stimulus to the detectors for calibration.

The outputs of the preamp go to unipolar shaping amplifiers with a shaping time of approximately $2 \mu \mathrm{sec}$. These are 5 pole quasi-Gaussian filters and have gated baseline restorers. A simple differentiator and compensation network provide peak detection. The outputs 
of the amplifiers go to a set of discriminators whose output allows the digital electronics to control the data acquisition. The digital signals available are Peak Detection (signal active when the unipolar pulse reaches its peak), (LLD) Low Level Discriminator (signal is above a threshold set by the Digital Electronics), (ULD) Upper Level Discriminator (signal is above the acceptable range of the Analog/Digital converter), and Reset (active during a preamp reset). The LLD is also used by the timing logic in the Digital Electronics to provide simple pile-up rejection. Each channel has its own low power Analog/Digital converter (16 bit converter with 12 bits used by the system) controlled by the Digital Electronics.

The STE Digital Electronics takes the ADC signal in the form of converted pulse heights (energy) together with an event strobe and accumulates it, and provides the results to the IMPACT IDPU via a serial interface. The Digital Electronics is implemented in the STE Interface FPGA (SIF) Actel and an SRAM memory. The SIF also runs the SWEA instrument in the case of STE-D. The front end ULD, LLD, and Reset event rates are counted in the SIF. The SIF provides an event threshold energy level to the analog front end chain via a Digital to Analog Converter (DAC). This level is set by the IDPU and is commandable from the ground. These interfaces are replicated for each of the 4 detector segments.

The SIF also provides the test pulser input to the analog electronics for calibration. The test pulser has a fixed event rate and a programmable amplitude which can be set to ramp up smoothly or in 8 discrete levels. The pulser operation is set by the IDPU in response to ground commands.

The SIF controls the STE detector bias voltage over a range of $0-150 \mathrm{~V}$. Each of the 4 STE units has a separately programmed bias voltage (shared by the 4 detectors in the STE unit). The bias voltage is set by the IDPU in response to ground commands.

The SIF controls a protection circuit which identifies an over current event in the ana$\log$ front end (perhaps due to Single Event Latchup) and powers off the analog front end automatically. The IDPU can reset this circuit in response to a command from the ground.

The SIF also controls the STE door actuators. It applies power to the open or close actuators until either the end of travel sense switch is activated or a pre-programmed timeout occurs (to protect the actuator from over-heating). The door actuation is initiated by the IDPU either in response to a ground command or a spacecraft status change (e.g., a thruster firing warning).

The SIF determines when a valid event has occurred based on information from the analog front end, including the peak detect signal, the ULD, the LLD, and the Reset signals. The scheme is designed to eliminate pile-up and veto ULD events and events that occur during Reset. Once a valid event has been recognized the event is converted by the ADC and binned into an accumulator. The system can only deal with one event at a time, up to $\sim 300,000$ events/second combined for all 4 detectors. The system is designed to be nonpreferential so that one detector cannot lock out another.

Event binning is based on a programmable look-up table (LUT) that takes a combination of the 12-bit energy amplitude provided by the ADC and the 2-bit detector ID and generates an 8-bin accumulator ID to increment. The standard LUT generates 32 logarithmically spaced energy channels (in 1/v) for each of the 4 detectors (see Table 2). Other LUTs are used for calibration and diagnostics.

The events are accumulated for 2 seconds and then read out to the IDPU. The IDPU passes the 2-second resolution data (both spectra and rate counters) to the burst memory. It also averages the data for a programmable interval (nominally 10 seconds) for the normal telemetry channel, and longer (1 minute) for the beacon telemetry channel. 
Table 2 Nominal STE Energy Bins. The energy bins accumulate events from the next energy level below to the energy level indicated; bin "0" counts from the discriminator threshold level to the indicated level

\begin{tabular}{llrlllll}
\hline Bin & $\begin{array}{l}\text { Energy, } \\
\text { keV }\end{array}$ & Bin & $\begin{array}{l}\text { Energy, } \\
\text { keV }\end{array}$ & Bin & $\begin{array}{l}\text { Energy, } \\
\text { keV }\end{array}$ & Bin & Energy \\
\hline 0 & 2.00 & 8 & 6.00 & 16 & 11.21 & 24 & 26.99 \\
1 & 2.50 & 9 & 6.50 & 17 & 12.27 & 25 & 31.14 \\
2 & 3.00 & 10 & 7.00 & 18 & 13.50 & 26 & 36.32 \\
3 & 3.50 & 11 & 7.50 & 19 & 14.92 & 27 & 42.92 \\
4 & 4.00 & 12 & 8.08 & 20 & 16.58 & 28 & 51.49 \\
5 & 4.50 & 13 & 8.72 & 21 & 18.53 & 29 & 62.91 \\
6 & 5.00 & 14 & 9.45 & 22 & 20.84 & 30 & 78.60 \\
7 & 5.50 & 15 & 10.27 & 23 & 23.62 & 31 & 100.0 \\
\hline
\end{tabular}

\section{Mechanical}

On each STEREO spacecraft there are separate STE-U and STE-D arrays with FOVs aligned with the nominal Parker spiral magnetic field direction facing toward $\left(45^{\circ}\right)$ and away $\left(225^{\circ}\right)$ from the Sun, respectively. Since the two spacecraft are rotated by $180^{\circ}$ about the sunspacecraft line relative to each other, the STE FOVs are also rotated by $180^{\circ}$ between spacecraft. The total field of view (FOV) of the STE-D is $80^{\circ} \times 80^{\circ}$, while that of the STE-U is restricted to $70^{\circ} \times 70^{\circ}$ for sunshade clearance. The $1 \times 4$ linear arrays of $3 \mathrm{~mm} \times 3 \mathrm{~mm}$ SSDs are mounted on a ceramic circuit board inside an aluminum housing (Fig. 1). A goldplated $\mathrm{BeCu}$ grid (Fig. 3, top) covering the opening serves as a Faraday cage to suppress the electric field of the SSD high voltage. STE has a reclosable door (Fig. 3, top) to protect the SSDs during ground handling, launch, and maneuvers (thruster plumes). Space constraints and the wide temperature range made traditional actuators problematic, so an SMA (Shape Memory Alloy, typically about equal parts $\mathrm{Ti}$ and $\mathrm{Ni}$ ) actuated device was designed instead (Fig. 3, bottom). SMAs contract when heated above their transition temperature, and can be trained to return to their original shape when cooled. For this application, about $10^{\prime \prime}$ of $0.003^{\prime \prime}$ dia. Flexinol wire (manufactured by Dynalloy, Inc.) was used as an actuator for each direction. The linear wire contraction is converted into a rotary stroke of $120^{\circ}$ by wrapping the free wire ends around tensioners attached to the door shaft. In order to fit this length of wire in the available mechanism volume of $1.6^{\prime \prime} \times 2^{\prime \prime} \times 38^{\prime \prime}$ inches, the wire was wrapped around a series of pulleys to fold the required length into the available space.

All moving plastic parts were made from Vespel SP, a $\mathrm{MoS}_{2}$ impregnated polyimide with a very low coefficient of friction in vacuum. The toroidal door shaft bearings are synthetic sapphire, which is very hard and has a fairly low coefficient of friction when dry. The 303 stainless steel door shaft was coated with Ti nitride and dry lubed using a process called Brycoat. This consists of impregnating the surface with $\mathrm{MoS}_{2}$. Knowledge of the door position was provided by custom designed very low actuation force end-of-travel (EOT) switches

To move the door, $9 \mathrm{~V}$ is applied to the appropriate SMA wire. The wire resistively dissipates $1.6 \mathrm{~W}$, self-heating it to the transition temperature and contracting the wire, and pulling the door either open or closed. The door is held in the cam detent until enough force is generated in the wire to overcome the overcenter plunger, and the door snaps smartly into the opposite position. As the door begins to move, one EOT switch opens, indicating the beginning of travel, and once it reaches its end of stroke the other EOT switch closes and shuts off the current. 

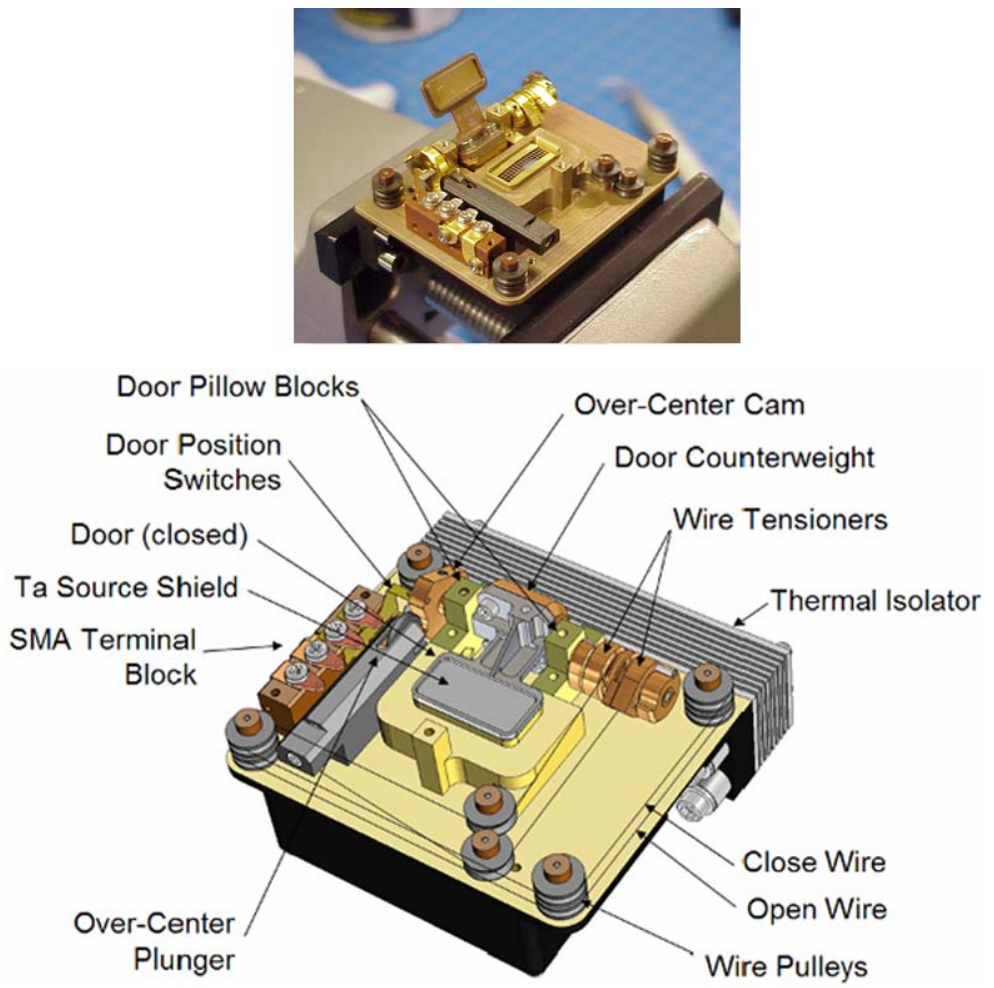

Fig. 3 Top: STE detector housing showing door open. Bottom: Schematic showing reclosable door mechanism

For the sunward-looking STE-U, a sun shield keeps sunlight and glint from the instrument aperture (Fig. 4). The sun shield has a double-beveled edge so that any glint from the sunward-most edge is blocked from entering the aperture by the second edge, and the second edge is prevented from generating glint by the shadow of the first edge.

The STE SSDs are passively cooled to operate in the range of $-30^{\circ} \mathrm{C}$ to $-90^{\circ} \mathrm{C}$ on orbit to minimize electrical noise. The SSDs and housing (with sun shield) are separated by PEEK thermal isolators (Fig. 3) from the preamp for STE-U, and from the SWEA instrument for STE-D. The other major heat path is through the approximately thirty 30ga. wires. Custom drawn and Teflon coated Manganin (Cu86/Mn12/Ni2) wires, with less than half the thermal conductivity of copper, were used for this application. Silver Teflon tape was applied to sunward facing surfaces to reflect sunlight during off-pointing maneuvers, preventing excessive heating of the electronics. As the STE-D instrument is mounted far from the spacecraft and in its shadow, the majority of its outer surface is gold alodine to prevent it from getting too cold. The STE-U is on the sunward side and sitting in direct sunlight, necessitating the thermally isolated sunshade and extensive silver Teflon tape and black paint (Fig. 4). Post-launch the SSDs reached equilibrium temperatures of $-63^{\circ} \mathrm{C}$ and $-80^{\circ} \mathrm{C}$ for STE-D, and $-38^{\circ} \mathrm{C}$ and $-51^{\circ} \mathrm{C}$ for STE-U, for spacecraft A and B, respectively.

The electrostatic cleanliness (ESC) requirements necessitate careful control of outer skin charging by use of conductive materials on most exposed surfaces, particularly those in shadow. The silver Teflon tape used on the STE instruments as well as on the sunward facing spacecraft blankets was ITO (Indium-Tin-Oxide) sputtered to provide a path for draining any 


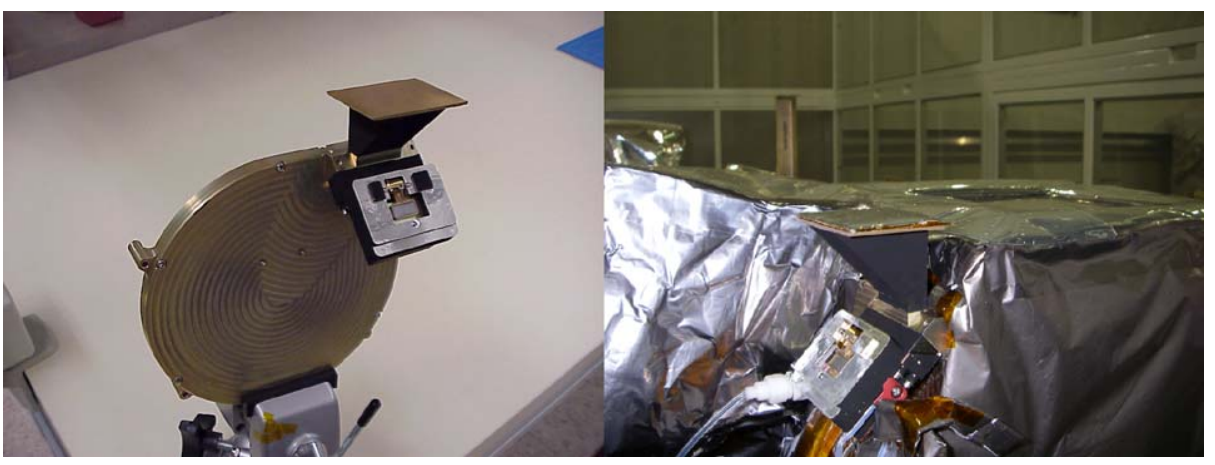

Fig. 4 STE-U sunshield (left) and thermal blanketing (right)

charge that develops due to photoemission or other effects. The STE-U preamp and SWEA electronics are covered with MLI blankets with conductive black Kapton outer surfaces with a Ge coating for improved thermal properties. To prevent leakage of electric fields from electronics, all mechanical joints in the STE and preamp housings were staggered, and an aperture grid covered the SSDs.

\section{Calibration}

Calibrations of the STE instruments were performed in the laboratory using radioactive sources and an electron gun. Figure 5 shows a typical spectrum for a STE detector exposed to a ${ }^{55} \mathrm{Fe}$ and a ${ }^{109} \mathrm{Cd}$ radioactive sources simultaneously, taken at room temperature. The ${ }^{55} \mathrm{Mn} \mathrm{K}_{\alpha} \mathrm{X}$-ray at $5.9 \mathrm{keV}$ is the largest peak in the spectrum and the $3 \mathrm{keV}$ peak from the daughter isotope ${ }^{109} \mathrm{Ag}$ is clearly evident. The broad peaks centered around $68 \mathrm{keV}$ and $40 \mathrm{keV}$ are due to the 84 and $62 \mathrm{keV}{ }^{109} \mathrm{Cd}$ conversion electrons that have lost energy in the source and detector Windows. A similar ${ }^{109} \mathrm{Cd} /{ }^{55} \mathrm{Fe}$ radioactive source on the inside of the STE reclosable door provides in-orbit calibration of the SSDs.

The primary calibration involves measuring the response of the STE SSDs to incident electrons of known energy from an accelerator. Photo-electrons generated by a UV lamp illuminating a specially coated photo-anode are accelerated through a potential drop to produce a wide $(\sim 3 \mathrm{~cm})$ parallel mono-energetic electron beam with energy adjustable from 0 to $30 \mathrm{keV}$. A Helmholz coil was positioned outside the chamber to nullify the Earth's magnetic field and minimize the curvature of low energy electrons.

In addition, an on-board Test Pulser that runs at $\sim 2000 \mathrm{c} / \mathrm{s}$ with a ramping amplitude, cycling every 40 seconds, is used to stimulate the preamplifier and downstream electronics chain and verify the stability and linearity of the electronics. It has two modes; smoothly ramping (good for testing ADC DNL), and stepping over 8 linearly spaced discrete amplitudes (for calibrating front end gain and electronic noise).

Figure 6 shows the pulse height distribution of one SSD in the STE instrument for normally incident $4 \mathrm{keV}$ electrons. The largest peak is observed with center energy at the STE ADC channel 18.4, along with an overlying Gaussian fit showing a FWHM of $1.1 \mathrm{keV}$. The low energy excess below the peak is due to the roughly 5 to $10 \%$ of electrons incident upon the SSD that scatter back out of the detector, resulting in partial energy deposition. 


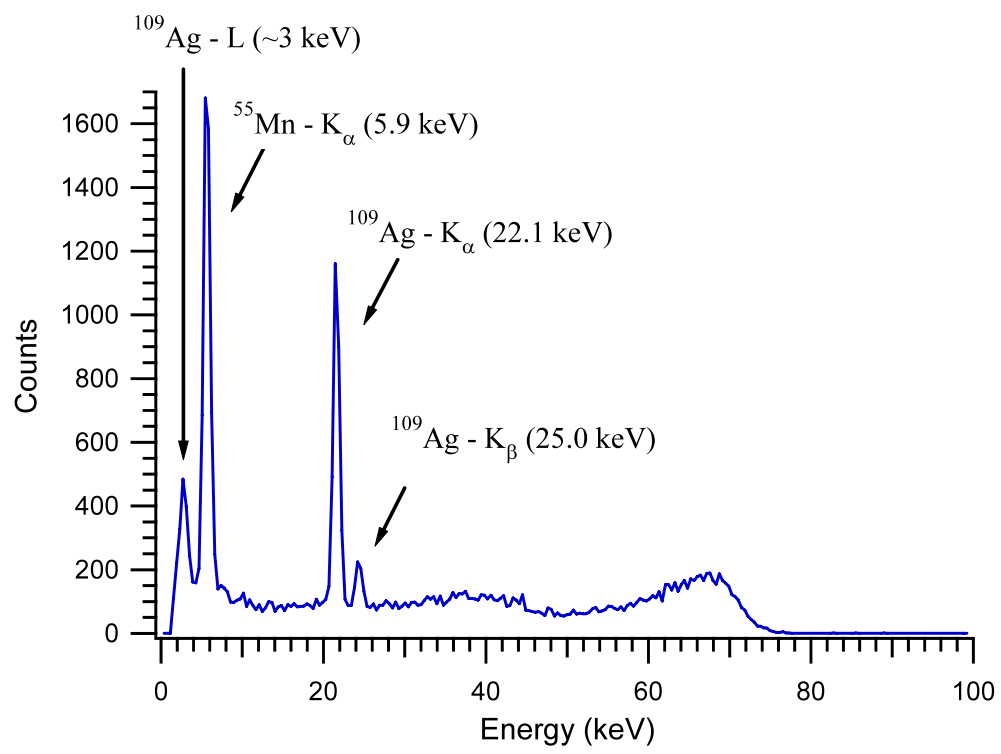

Fig. 5 Spectrum obtained by illuminated a STE detector at ambient temperature with a source of one $\mu \mathrm{Ci}$ ${ }^{55} \mathrm{Fe}$ and a one $\mu \mathrm{Ci}{ }^{109} \mathrm{Cd}$ simultaneously. The integration time is not sufficient to show the $88 \mathrm{keV}$ gamma emission of the ${ }^{109} \mathrm{Cd}$. The broad peaks centered around $68 \mathrm{keV}$ and $40 \mathrm{keV}$ are due to the 84 and $62 \mathrm{keV}$ ${ }^{109} \mathrm{Cd}$ conversion electrons that have lost energy in the source and detector Windows. A combination of ${ }^{109} \mathrm{Cd}$ and ${ }^{55} \mathrm{Fe}$ radioactive source to the inside of the door provides in-orbit calibration of the SSDs when door is closed
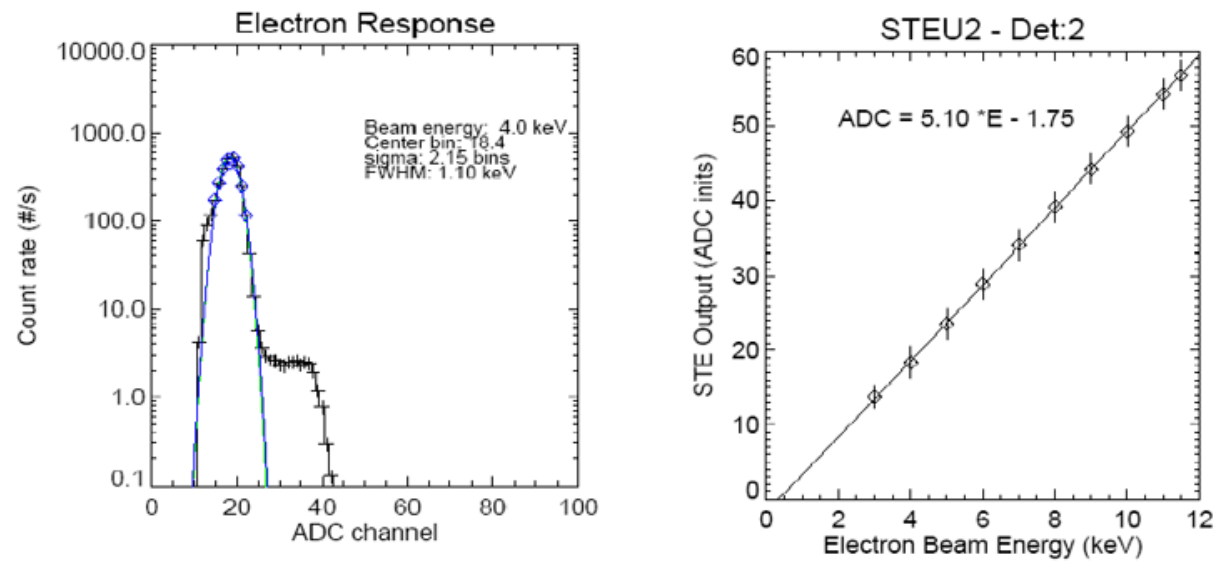

Fig. 6 Left panel: Response of a STE detector to a normally incident beam of $4 \mathrm{keV}$ electrons. The fit to the peak gives $\sim 1.1 \mathrm{keV}$ FWHM energy resolution. The secondary peak at twice the energy is due to pulse pileup from two electrons striking the detector simultaneously, unusually prominent because the beam is pulsed. Right panel: STE ADC channel output for different energy electrons (vertical error bars dominated by FWHM width of the detector response)

The STE sensor was mounted on a 3-axis manipulator to allow the instrument response to be determined as a function of the 2 incident angles and linear position along to the beam 


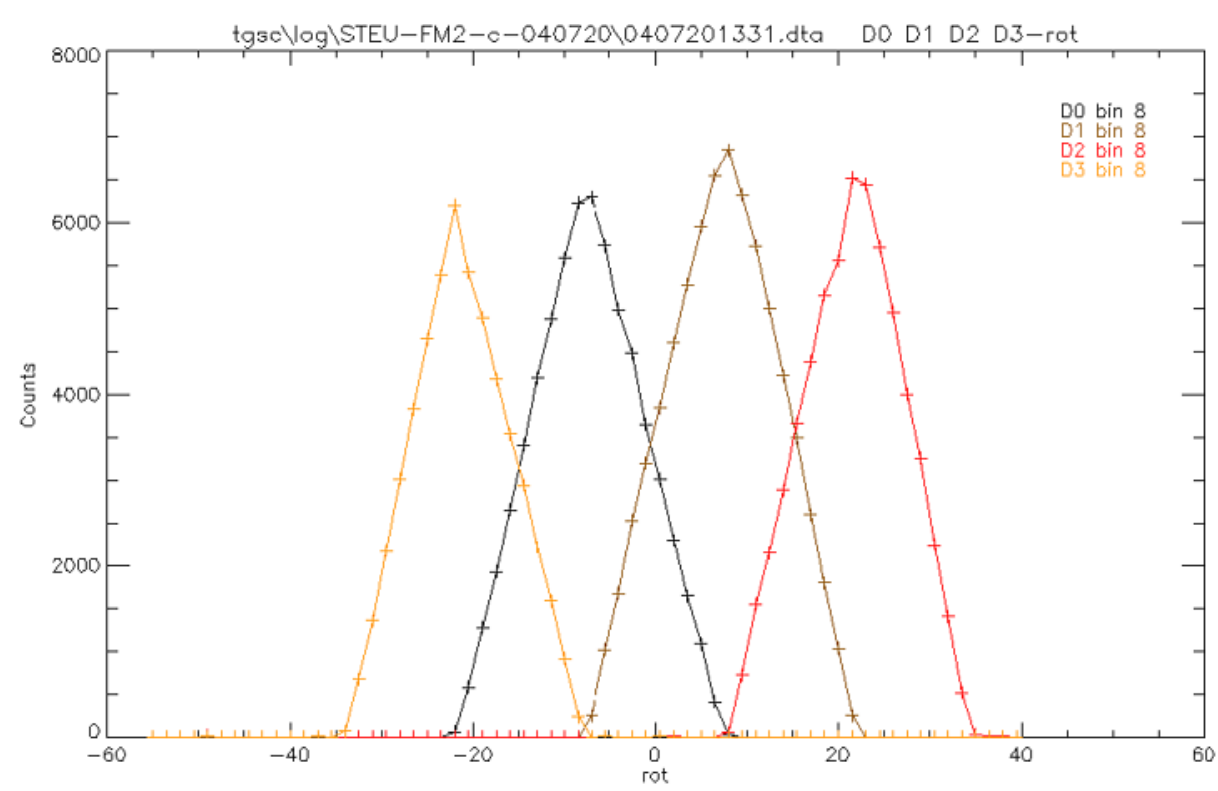

Fig. 7 Angular response in azimuth angle $\left(0^{\circ}\right.$ is normal incident to the detector plane $)$ for the four detectors of the STE-U sensor

width. Figure 7 shows the total count rate observed in each of the four pixels of STE-U as a function of the azimuth angle. This measurement was performed while keeping the electron beam energy constant at $10 \mathrm{keV}$.

For comparison, Fig. 8, left panel, shows preliminary observations of an impulsive solar electron event, and Fig. 8, right panel, the energy spectra for both the pre-event background and for the peak flux at each energy for the event, from STE-D for the B spacecraft. The lowest energy point is at $2 \mathrm{keV}$, and shows some detector noise as expected. This is an active time when the pre-event background is dominated by solar electron fluxes.

\section{Modes of Operation \& Data Format}

The normal mode of operation for STE is door open, test pulser off, with the energy Look Up Table (LUT) number zero for the nominal case (Table 2) in use, and normal telemetry time resolution set to 10 seconds. The Bias voltage is set to optimize the detector noise performance and the LLD threshold is set to give $<10 \mathrm{c} / \mathrm{s}$ background in the lowest energy channel. Note that the energy levels in Table 1 are nominal, depending on the gain of the front end.

The STE door will be closed periodically during the mission when the observatory is off-pointing or firing thrusters. Then, the SSDs are exposed to the ${ }^{109} \mathrm{Cd} /{ }^{55} \mathrm{Fe}$ radioactive source for calibration of the detectors and the front end gain and noise characteristics (peak width). The STE door calibration is often used with a special LUT to provide 256 linearly spaced energy bins to a single detector.

The STE Rates packet includes information on the state of the STE door, STE test pulser, accumulation time, and LUT in use. The LUT in use is encoded as the "STE Mode ID". Values 1-6 are used for various diagnostic modes used with the STE door source and test 

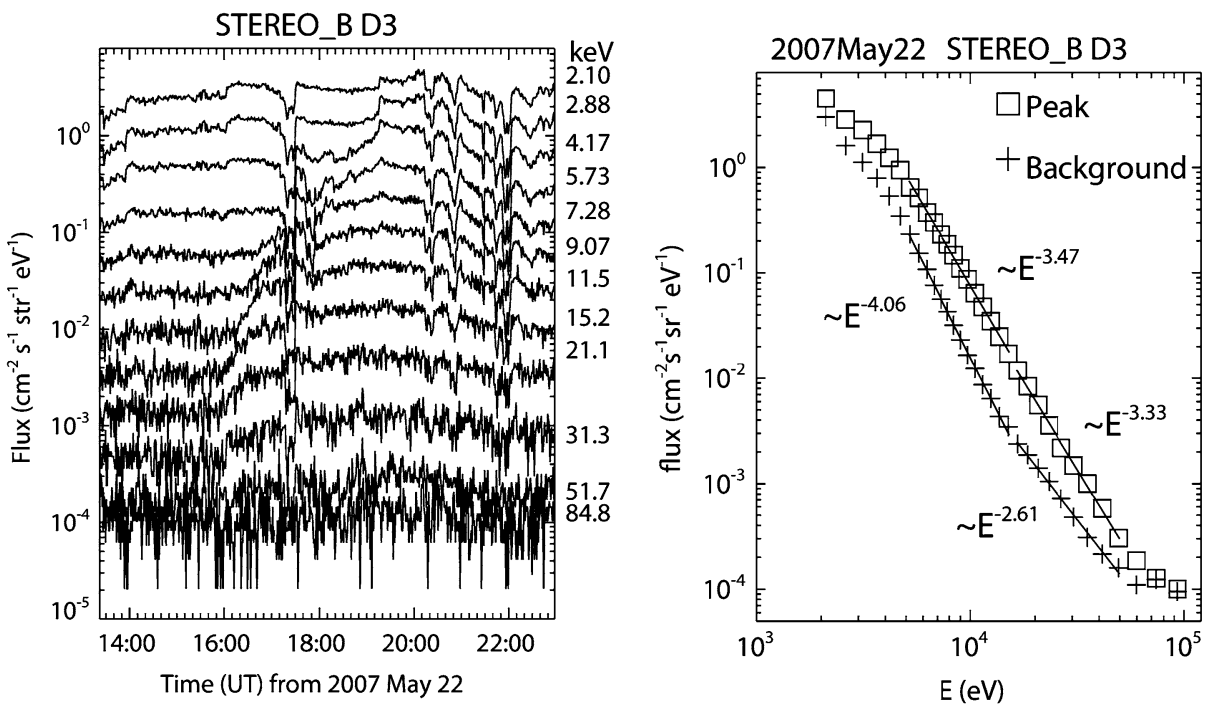

Fig. 8 Left panel: One-minute average electron fluxes observed downstream by a single STE detector on 2007 May 22. A solar energetic electron event begins at $\sim 1555$ UT, with velocity dispersion evident down to $\sim 3 \mathrm{keV}$. Right panel: Energy spectra from the same detector. The squares indicate the peak fluxes observed in the 32 energy bins, and the crosses show the pre-event electron fluxes

pulser. Other values may be used if new LUT are loaded post-launch. Threshold and detector HV settings are included in the STE parameter table that is transmitted periodically (once an hour).

The STE data is accumulated into telemetry packets. Details of these packets are described in the IMPACT Command and Telemetry database. Spectra packets contain the accumulated spectra data. Each energy bin count is log-compressed to 8-bits. In normal mode, a Spectra packet contains all the accumulated spectra for STE-U and STE-D (32 energies time 4 detectors times two STE units $=256$ counters per spectra packet).

Rates packets contain accumulated rate counters. Each counter is log-compressed to 8 bits. Each Rates packet contains 10 samples of LLD, ULD, and Reset counters for 4 detectors for each STE unit. The 10 samples are consecutive measurements at the accumulation time interval (nominally 10 seconds). The Rates packets also contain various detector status information. The format of burst and normal telemetry packets are the same, but with different packet identifiers. The difference is that Burst data has 2 -second resolution, while normal telemetry has 10 second resolution. Burst data is being taken continuously. A burst can be triggered by an increase in STE count rate, or by other IMPACT instruments-MAG or SWEA — or by the PLASTIC instrument. When a trigger occurs, five minutes of pre-trigger data is captured and stored, together with five minutes following the trigger.

STE Beacon data is a subset of the IMPACT Boom Suite Beacon packet produced once a minute. It contains 5-energy spectra (obtained by summing the 32-energy normal telemetry spectra into bands). Spectra are summed over detectors into 3 categories: STE-D, STE-U sunward detector, and the rest of STE-U. Beacon data also contains the combined STE-U and STE-D LLD rate. All of this is averaged over a 1-minute interval.

Acknowledgements We acknowledge the support of many colleagues, including B. Ludewigt, M. Colby, P. Berg. This research is supported in part by NASA Contract NAS5-00133 for IMPACT. 


\section{References}

S.D. Bale, P.J. Kellogg, K. Goetz, S.J. Monson, Transverse z-mode waves in the terrestrial electron foreshock. Geophys. Res. Lett. 25, 9-12 (1998)

S.D. Bale, M.J. Reiner, J.-L. Bougeret, M.L. Kaiser, S. Krucker, D.E. Larson, R.P. Lin, The source region of an interplanetary type II radio burst. Geophys. Res. Lett. 26, 1573-1576 (1999)

H.V. Cane, W.C. Erickson, Energetic particle propagation in the inner heliosphere as deduced from lowfrequency $(<100 \mathrm{kHz})$ observations of type III radio bursts. J. Geophys. Res. 108, 1203 (2003)

R.E. Ergun et al., Wind spacecraft observations of solar impulsive electron events associated with solar type III radio bursts. Astrophys. J. 503, 435-445 (1998)

J.T. Gosling et al., Solar electron bursts at very low energies: Evidence for acceleration in the high corona? Geophys. Res. Lett. 30(13), 1697-1700 (2003)

D.K. Haggerty, E.C. Roelof, Impulsive near-relativistic solar electron events: Delayed injection with respect to solar electromagnetic emission. Astrophys. J. 579, 841-853 (2002)

S. Holland, Fabrication of detectors and transisitors on high-resistivity silicon. Nucl. Instrum. Methods Phys. Res. A275, 537-541 (1989)

S.E. Holland et al., Development of low noise, back-side illuminated silicon photodiode arrays. IEEE Trans. Nucl. Sci. 44, 443-447 (1997)

Kaiser et al., The STEREO Mission: An Introduction. Space Sci. Rev. (2007, this issue). doi:10.1007/ s11214-007-9277-0

S. Krucker et al., On the origin of impulsive electron events observed at 1 AU. Astrophys. J. 519, 864-875 (1999)

D.E. Larson, R.P. Lin et al., Tracing the topology of the October 18-20, 1995, magnetic cloud with $\sim 0.1-$ $10^{2} \mathrm{keV}$ electrons. Geophys. Res. Lett. 24, 1911 (1997)

R.P. Lin, Energetic solar electrons in the interplanetary medium. Sol. Phys. 100, 537-561 (1985)

R.P. Lin, Observations of the 3D distributions of thermal to near-relativistic electrons in the interplanetary medium by the Wind spacecraft, in Coronal Physics from Radio and Space Observations, ed. by G. Trottet. Lecture Notes in Physics, vol. 483 (Springer, Berlin, 1997), p. 93

R.P. Lin, W.K. Levedahl, W. Lotko, D.A. Gurnett, F.L. Scarf, Evidence for nonlinear wave-wave interactions in solar type III radio bursts. Astrophys. J. 308, 954 (1986)

R.P. Lin et al., A three-dimensional plasma and energetic particle investigation for the wind spacecraft. Space Sci. Rev. 71, 125-153 (1995)

R.P. Lin et al., Observation of an impulsive solar electron event extending down to $\sim 0.5 \mathrm{keV}$ energy. Geophys. Res. Lett. 23, 1211-1214 (1996)

R.P. Lin, B.R. Dennis, G.J. Hurford et al., The Reuven Ramaty High-Energy Solar Spectroscopic Imager (RHESSI). Sol. Phys. 210, 3-32 (2002)

J.G. Luhmann et al., STEREO IMPACT investigation goals, measurements, and data products overview. Space Sci. Rev. (2007, this issue). doi:10.1007/s11214-007-9170-X

D. Maia, M. Pick, Astrophys. J. 609, 1082-1097 (2004)

E. Mobius, D. Rucinski, M.A. Lee, P.A. Isenberg, Decreases in the antisunward flux of interstellar pickup $\mathrm{He}+$ associated with radial interplanetary magnetic field. J. Geophys. Res. 10, 257 (1998)

D.V. Reames et al., Solar ${ }^{3} \mathrm{He}$-rich events and nonrelativistic electron events-A new association. Astrophys. J. 292, 716-724 (1985)

Sauvaud et al., Space Sci. Rev. (2007, this issue). doi:10.1007/s11214-007-9174-6

G.M. Simnett et al., The acceleration and release of near-relativistic electrons by coronal mass ejections. Astrophys. J. 579, 854-862 (2002)

C.S. Tindall, N.P. Palaio, B.A. Ludewigt, S.E. Holland, D.E. Larson, D.W. Curtis, S.E. McBride, T. Moreau, R.P. Lin, V. Angelopoulos, Silicon detectors for low energy particle detection. Trans. Nucl. Sci. (2008, to be submitted)

L. Wang, R.P. Lin, S. Krucker, J.T. Gosling, Evidence for double injections in scatter-free solar impulsive electron events. Geophys. Res. Lett. 33, L03106 (2006a). doi:10.1029/2005GL024434

Y.-M. Wang, M. Pick, G.M. Mason, Coronal holes, jets, and the origin of ${ }^{3} \mathrm{He}$-rich particle events. Astrophys. J. 639, 495 (2006b) 